\title{
Trabalho infantil em Pelotas: perfil ocupacional e contribuição à economia
}

\author{
Child labor in Pelotas: occupational \\ characteristics and contribution to the economy
}

Luiz Augusto Facchini 1

Anaclaudia Gastal Fassa 1

Marinel Dall'Agnol 1

M aria de Fátima Santos M aia 1

\footnotetext{
1 Programa

de Pós-Graduação

em Epidemiologia

Departamento de M edicina

Social, Universidade Federal de Pelotas,

Av. Duque de Caxias, 250/ 30 andar, 96030-001, Pelotas RS

Ifachini@terra.com.br
}

Abstract Child labor is a huge worldwide problem, particularly in developing countries. It is estimated that there are 352 million economic active children and teens younger than 18 years- old in the world, including not only paid work but also unpaid, illegal and informal work. In Brazil there are 9.3 million child workers between 10 and 17 years- old. There are few studies evaluating the children economic contribution to the family income. This paper presents the children and teens occupational characteristics and their economic contribution emphasizing its particularities by gender, age, school attendance and familiar socioeconomic status in a sample of 4.924 subjects between 6 and 17 years-old, representative of the low-income areas of Pelotas. Children and teens contributed in average with $18 \%$ of the family income. A mong workers, half of them contributed with at least $10 \%$ of the family income and one forth contributed with $25 \%$ or more. The proportion of the children and teens contribution to the total family income, the non-attendance to school among teens workers and the number of hours of children and teens work increase with the decrease of the adult family income.

Key words Child labor, O ccupational profile, Economic contribution
Resumo 0 trabalho infantil é um fenômeno global de grandes proporções, especialmente em países em desenvolvimento. Estima-seque, em todo o mundo, 352 milhões de crianças e adol escentes menores de 18 anos são economicamenteativos, incluindo atividades remuneradas, trabalho não- pago, ilegal e no setor informal da economia. No Brasil, cerca de 9,3 milhões de crianças entre 10 e 17 anos trabaIham. Os estudos sobre a contribui ção econômica das crianças à renda familiar são escassos. Este artigo apresenta o perfil ocupacional e a contribui ção econômica de crianças e adolescentes, com destaque para aspectos relacionados à idade, ao gênero, à escolaridade e à situação socioeconômica de suas famílias em uma amostra de 4.924 indivíduos entre 6 e 17 anos, representativa dos setores urbanos pobres de Pelotas. As crianças e adolescentes trabalhadores contribuíam em média com 18\% da renda familiar. Dos que trabalhavam, metade contribuía no mínimo com $10 \%$ da renda familiar e um quarto respondia por $25 \%$ ou mais da renda familiar. Quanto menor a renda familiar dosadultos, maior a proporção da contribui ção de crianças e adol escentes à renda familiar total, maior a exclusão escolar de adolescentes trabalhadores e maior a jornada de trabalho infanto-juvenil.

Palavras-chave Trabalho infantil, Perfil ocupacional, Contribuição econômica 


\section{Introdução}

0 trabalho infantil é um fenômeno global de grandes proporções, principalmente em países em desenvolvimento, onde vivem mais de $96 \%$ das crianças e adolescentes trabalhadores (ILO, 2002). A luta contra o trabal ho infantil tem crescido na última década, mostrando avanços importantes, em vários países do mundo (ILO, 2002). No Brasil, a prevalência de trabalho infantil mostrou uma redução de $30 \%$ no período, boa parte devido aos programas de erradicação e prevenção do problema e a uma maior consciência social dos direitos de crianças e adolescentes (IBGE, 2001). Ainda assim, estima-se que, em todo o mundo, 352 milhões de crianças e adolescentes menores de 18 anos são economicamente ativos, incluindo atividades remuneradas, mas também trabalho não pago, ilegal e no setor informal da economia (ILO, 2002). N esta população, $88 \%$ das crianças de 5 a 14 anos e $42 \%$ dos adolescentes de 15 a 17 anos trabalham em atividades inadequadas para a idade, que precisam ser eliminadas em respeito a conven ções e padrões internacionais estabelecidos pela O rganização Internacional do Trabalho e firmadas por muitos países, incluindo o Brasil (ILO, 1998 e 2002). No país, cerca de 9,3 milhões de crianças entre $10 \mathrm{e}$ 17 anos trabalham, situação que alcança meio milhão de gaúchos desta faixa etária (Comissão Estadual de Combate ao Trabal ho Infantil no Rio Grande do Sul, 1996; Cruz N eto et al., 1998). Em termos relativos, o trabalho é uma realidade cotidiana para $25 \%$ da população brasileira entre 10 e 17 anos de idade (U nicef, 1996).

A magnitude do problema e a multiplicidade de riscos decorrentes da exploração do trabalho infantil estão demandando ações efetivas de governos e sociedades (ILO, 1999 e 2002). Infelizmente, a extensão e a natureza do impacto do trabalho infantil na saúde, educação e desenvolvimento das crianças tem sido relativamente pouco investigada (Scanlon et al., 2002). Ainda assim, as evidências indicam que seu impacto pode ser mais prejudicial quanto menor a idade da criança, maior a interferência em atividades escolares, recreação e repouso, maior a jornada de trabalho, incluindo o trabalho noturno, e maior a exposição a riscos ocupacionais (Fassa et al., 2000).

Os estudos populacionais que investigam a extensão do trabalho infantil, seus aspectos econômicos e suas conseqüências sociais também são raros ( Lim, 2000). As estatísticas nacionais sobre população e força de trabalho estão crescentemente disponíveis na maioria dos países do mundo, através de censos e pesquisas de amostras específicas de trabalhadores, mas dados sobre trabalho infantil raramente são coletados através destas fontes, que não captam atividades econômicas em menores e aquelas chamadas de informais ou invisíveis (ILO, 2000).

Portanto, para conhecer o problema do trabalho infantil e suas conseqüências, particularmente de suas piores formas, necessita-se do incremento de pesquisas que proporcionem diagnósticos abrangentes, bases de dados de boa qualidade, monitoramento periódico, avaliação de mudanças no mundo do trabal ho e identificação de soluções inovadoras e efetivas (Graitcer et al., 1998; U nicef, 1997). M as as pesquisas sobre trabalho infantil apresentam muitos desafios. São grandes as dificuldades em obter informações de crianças, famílias e empregadores sobre a inserção infantil no trabaIho, as tarefas que realizam, quanto ganham, qual o destino dos recursos obtidos e quais os problemas decorrentes da transformação de crianças em trabalhadores (ILO, 2000). Talvez esta seja uma das razões para a escassez de estudos sobre a contribuição econômica das crianças à renda familiar. Os poucos estudos disponíveis geralmente abordam pequenas amostras e os resultados não são estratificados por idade, sexo, tipo e tamanho da família ( $\mathrm{Hi}$ mes et al.,1994). Entretanto, estes estudos mostram que as crianças geral mente contribuem com uma substancial proporção da renda familiar, mesmo considerando realidades como a da América Latina, onde os rendimentos infantis são bastante baixos (Anker et al., 1995).

Buscando contribuir para o conhecimento do problema em nosso meio, investigou-se a prevalência do trabalho infantil e suas relações com a educação e a saúde de crianças e adolescentes em uma amostra de cerca de 5.000 indivíduos entre 6 e 17 anos e 3.000 famílias, representativa dos setores urbanos pobres de Pelotas. 0 estudo detal ha a caracterização das diversas formas de uso da força de trabalho infantil e a utilização dos recursos produzidos pelas crianças para a economia familiar elocal, em um contexto de marcante desindustrialização, aumento do desemprego e do trabalho informal.

0 presente artigo apresenta o perfil ocupacional e a contribuição econômica de crianças e adolescentes entre 6 e 17 anos, com desta- 
que para aspectos relacionados à idade, ao gê nero, à escolaridade eà situação socioeconômica de suas famílias.

\section{Metodologia}

Estudou-se o trabalho infantil em Pelotas através de delineamento epidemiológico transversal de base populacional (Kleinbaum et al., 1982; Rothman et al., 1998). Este artigo detal ha os aspectos metodológicos referentes à caracterização do trabalho infantil e sua contribuição econômica. Os aspectos referentes à saúde e educação têm sido apresentados em outras publicações (Facchini et al., 2003).

Considerou-se trabalho infantil qualquer atividade laborativa regular, remunerada ou não, domiciliar ou não. A definição capta as atividades infantis de auxílio às demandas domésticas (cuidado da casa ou de irmãos menores), o trabal ho formal remunerado (comerciários) e inclusive atividades informais (catadores de papéis, guardadores de carros). Caracterizou-se 0 trabalho no dia da entrevista e o trabal ho pré vio. U tilizou-se a informação sobre o trabalho atual para traçar o perfil ocupacional e estimar a contribuição financei ra de crianças e adolescentes à economia familiar e do município. Caracterizou-se a inserção ocupacional de todos os membros da família e sua participação na renda familiar, para estimar com maior precisão a contribuição financeira das crianças trabalhadoras. As informações sobre o trabal ho doméstico realizado por crianças e adolescentes no próprio domicílio não foram incluídas neste artigo, tanto porque na grande maioria das vezes não são atividades remuneradas, quanto devido a suas enormes particularidades, que serão exploradas em outra publicação.

A população do estudo é constituída por famílias residentes em setores urbanos populares de Pelotas. Estimou-se uma amostra de indivíduos entre 6 e 17 anos de idade, tomandose como referência uma prevalência de trabaIho infantil em torno de $10 \%$ e um problema de saúde de baixa prevalência (3\%) em não trabalhadores. Adicionaram-se $15 \%$ para controle de fatores de confusão e $10 \%$ para controle de perdas, totalizando 4.390 indivíduos, amostra suficiente para os propósitos deste artigo.

$\mathrm{Na}$ época do estudo, Pelotas tinha uma população urbana de 265.192 habitantes, uma média de 3,5 pessoas por domicílio e $23 \%$ de indivíduos na faixa etária estudada (IBGE,
1992). Estimou-se necessário visitar cerca de 6.000 domicílios para localizar a amostra. Dentre os 70 setores populares, sortearam-se aleatoriamente 22, visitando-se todos os domicílios em cada setor. Os setores populares foram identificados como aqueles com uma proporção menor do que 1,5\% dos chefes de família recebendo 20 salários mínimos ou mais (IBGE, 1992). Todos os indivíduos ente 6 e 17 anos residentes em cada domicílio eram elegíveis para 0 estudo.

Os dados foram obtidos entre janeiro e junho de 1998, por 24 estudantes de medicina e de enfermagem, especialmente treinados para o estudo. 0 treinamento capacitou os auxiliares para a coleta acurada dos dados e para compreender a complexidade da investigação. Utilizaram-se mapas detal hados dos setores para orientar os entrevistadores. Obtido o consentimento informado da dona da casa em participar do estudo, um auxiliar de pesquisa entrevistava a mãe e outro auxiliar entrevistava a criança. Todos os domicílios visitados no setor e os questionários real izados foram registrados em uma folha de conglomerados.

Os questionários foram revisados, identificando-se e corrigindo-se questões não respondidas, respostas e codificações mal registradas. Este processo ocorreu durante o trabalho de campo, facilitando a busca de informação em falta. Os problemas observados foram registrados em uma planilha e discutidos semanalmente com a equipe e o entrevistador. Cerca de $5 \%$ dos questionários foram parcial mente refeitos por supervisores treinados, verificandose a qualidade dos dados.

O processamento dos dados incluiu a codificação das questões fechadas, a tabulação das questões abertas e sua codificação, a revisão dos códigos, sua dupla digitação e a correção da versão final da base de dados. A análise dos dados está sendo realizada através dos softwares SPSS for W indows 6.1 (SPSS Inc., 1997; Dean et al., 1995). Identificou-se o perfil de trabalho de crianças e adolescentes e sua contribuição à economia familiar e do município, tanto através de sua descrição, quanto de sua estratificação segundo gênero, grupo etário e nível socioeconômico.

\section{Resultados}

Num total de 3.171 domicílios localizados em 22 setores censitários de baixa renda, foram en- 
trevistados 4.924 indivíduos entre 6 e 17 anos de idade. As perdas foram de 7,5\%. Dos entrevistados, $51 \%$ eram do sexo masculino, $76 \%$ eram brancos e $95 \%$ estudavam. Cerca de $21 \%$ (1.014) já haviam trabalhado alguma vez; 9,7\% (476) estavam trabalhando no momento da entrevista; e 3,4\% (169) procuravam trabalho.

Em relação à idade das 476 crianças trabaIhadoras, 70\% tinham entre 14 e 17 anos de idade, $25 \%$ entre 10 e 13 anos, 5,3\% entre 6 e 9 anos e $88 \%$ estavam no mercado informal. Q uanto ao gênero, 71\% eram meninos e 29\%, meninas ( $p<0,001 ; R P=2.4$; IC $95 \% 1.9-2.8)$. Dos 14 aos 17 anos, 10\% dos meninos e 17\% das meninas trabal havam no mercado formal $(p=0,02 ; R P=1.23 ; I C 95 \%=1.0-1.5)$.

A prevalência de trabalho infantil foi de $21 \%$ entre 14 e 17 anos; $7 \%$ entre 10 e 13 anos, e 1,5\% entre 6 e 9 anos. A taxa de ocupação entre os meninos foi de $2,3 \%$, de 6 a 9 anos de idade; $10 \%$, de 10 a 13 anos e $29 \%$, de 14 a 17 anos. Entre as meninas, de 6 a 9 anos de idade, $0,5 \%$ trabalhavam; de 10 a 14 anos, cerca de $4 \%$ estavam trabalhando e de 14 a 17 anos, 13\% trabal havam. A presença dos meninos no trabalho foi significativamente maior do que a das meninas em todas as faixas etárias, sendo a razão de prevalência (intervalo de confiança $95 \%)$, respectivamente, de $4,9(1,7-14,1)$ dos 6 aos 9 anos; $2,5(1,7-3,7)$ dos 10 aos 13 anose $2,3(1,9-2,8)$ dos 14 aos 17 anos.

Os ramos econômicos com maiores prevalências de trabal ho infantil foram comércio (34\%); serviços não domésticos (24\%); serviços domésticos (20\%); construção civil (13\%); e indústria (5\%). Em todas as faixas etárias, atividades em comércio e serviços domésticos foram significativamente mais freqüentes entre as meninas, enquanto atividades em indústria, construção civil e serviços não domésticos foram al tamente predominantes entre os meninos.

Servente de pedreiro ( $10 \%$ ), auxiliar em bares, mercearias e lanchonetes $(9,5 \%)$, vendedor (9,2\%), limpeza de pátios (6,5\%), babá (6\%) e empregada doméstica $(5,3 \%)$ foram as ocupações mais freqüentes na população estudada.

Cerca de $40 \%$ das crianças e adolescentes trabal havam 40 horas ou mais semanalmente e $16 \%$ trabal havam à noite mais de 10 horas por semana. Até os 13 anos de idade, $42 \%$ das meninas e $21 \%$ dos meninos trabal havam 40 horas ou mais ( $p=0.04)$. Entre 14 e 17 anos de idade, $43 \%$ dos meninos e meninas trabal havam 40 horas ou mais.
A renda média mensal das crianças e adolescentes trabalhadores $(n=476$ ) foi de $R \$ 72,00$, sendo $R \$ 27,00$ para crianças de 10 aos 13 anos de idade e $R \$ 91,00$ para adolescentes de 14 a 17 anos. M ensalmente, cerca de $40 \%$ daqueles que trabalhavam recebiam $\mathrm{R} \$ 60,00$ ou menos; $17 \%$ recebiam entre $R \$ 61,00$ e $R \$ 120,00 ; 18 \%$ recebiam mais de $R \$ 120,00,16 \%$ não recebiam pagamento em dinheiro e 7\% não informaram a renda. A prevalência de meninos (77\%) recebendo dinheiro pelo trabalho realizado foi significativamente maior que a de meninas $(65 \%)(p<0,05)$. Dos 6 aos 13 anos de idade, a proporção de meninos e meninas trabalhando sem remuneração não mostrou diferença significativa. Dos 14 aos 17 anos de idade, a proporção de meninas trabalhando sem remuneração monetária era duas vezes maior do que a de meninos ( $p<0.01 ; R R=2,1 ;$ IC $95 \%$ 1,2-3,7).

A diferença na remuneração do trabalho segundo o gênero, também está evidente na renda média mensal que era, respectivamente, de $R \$ 79,00$ para os meninos e $R \$ 54,00$ para as meninas $(p<0,001)$. Dos 14 aos 17 anos, a renda média mensal dos meninos era de $R \$ 102,00$ e das meninas de $R \$ 65,00$ ( $p<0,001)$. Dos 10 aos 13 anos, a proporção de meninos recebendo mais de $R \$ 60,00$ mensais era duas vezes maior do que a de meninas. Dos 14 aos 17 anos, a proporção de meninos (32\%) recebendo mais de $R \$ 120,00$ por mês era cerca de 3 vezes maior do que a de meninas $(11 \%)(p<0,01)$.

A prevalência de trabalho infantil não se mostrou associada à renda familiar dos adultos para o conjunto da população estudada. Entretanto, dos 10 aos 13 anos de idade, o trabal ho infantil foi significativamente maior em famílias cuja renda dos adultos era menor (Tabela 1). Não se observou uma variação significativa na renda média obtida por crianças e adolescentes, em função da renda familiar dos adultos.

As crianças e adolescentes trabalhadores contribuíam em média com $18 \%$ da renda familiar. Entretanto, metade daqueles que trabaIhavam contribuía no mínimo com $10 \%$ da renda familiar e um quarto dos trabalhadores menores respondia por $25 \%$ ou mais da renda familiar. Em média, a contribuição à renda familiar era de $3 \%$ dos 6 aos 9 anos de idade, $14 \%$ dos 10 aos 13 anos e $20 \%$ dos 14 aos 17 anos. A contribuição média dos meninos foi de $19 \%$ e das meninas, $15 \%(p=0,20)$. Até os 9 anos de idade, a proporção da renda familiar oriunda da renda infantil era similar para me- 
Tabela 1

Prevalência de trabalho infantil segundo a idade da criança e a renda familiar dos adultos. Pelotas, RS, 1998.

\begin{tabular}{|c|c|c|c|c|}
\hline \multirow[b]{2}{*}{ Renda dos adultos } & \multicolumn{4}{|c|}{ Prevalência de Trabalho/Idade } \\
\hline & $\begin{array}{r}\text { 6-9 anos } \\
(\mathrm{n}=1479)\end{array}$ & $\begin{array}{c}10-13 \text { anos } \\
(n=1474)\end{array}$ & $\begin{array}{c}14-17 \text { anos } \\
(n=1410)\end{array}$ & $\begin{array}{c}\text { total } \\
(n=4363)\end{array}$ \\
\hline menos de $3 \mathrm{sm}(\mathrm{n}=1718)$ & 1.1 & 9.1 & 20.4 & 9.5 \\
\hline 3 a $6 \mathrm{sm}(n=1426)$ & 1.3 & 5.8 & 18.9 & 8.5 \\
\hline mais de 6 sm $(n=1219)$ & 1.6 & 5.7 & 21.5 & 10.2 \\
\hline Total $(n=4363)$ & 1.3 & 7.1 & 20.3 & 9.4 \\
\hline $\begin{array}{l}\text { p-valor tendência linear } \\
\text { sm = salários mínimos }\end{array}$ & 0,53 & 0,02 & 0,80 & 0,80 \\
\hline
\end{tabular}

ninos e meninas. Dos 10 aos 13 anos, a contribuição dos meninos era de $15 \%$ e a das meninas, $12 \%$, diferença que se torna significativa dos 14 aos 17 anos, quando a contribuição dos meninos passava para $21 \%$ e a das meninas para $17 \%(p<0,001)$.

Q uanto menor a renda familiar dos adultos, maior a proporção da contribuição de crianças e adolescentes à renda familiar total. Assim, em famílias cuja renda dos adultos era menor do que 3 salários mínimos, $47.3 \%$ dos menores trabalhadores aportavam $25 \%$ ou mais da renda familiar total. Em contraste, nas famílias com renda dos adultos superior a 6 salários mínimos, apenas $1 \%$ dos jovens trabalhadores contribuíam com $25 \%$ ou mais da renda familiar total $(p<0,000)$ (Tabela 2).

Em famílias com crianças ou adolescentes trabalhando, a renda média familiar total era $\mathrm{R} \$ 746,00$, cerca de $9 \%$ maior do que a renda média de famílias em que não havia a ocorrência de trabalho infantil $(R \$ 691,00)$. Esta diferença de $R \$ 61,00$ representava um acréscimo médio anual de $\mathrm{R} \$ 732,00$ na renda de famílias cujos filhos menores de 18 anos trabal havam.

Independentemente da renda familiar dos adultos, uma maior proporção de meninas não contribuía monetariamente para a renda familiar, porque não recebia salário, e uma maior proporção de meninos contribuía com $25 \%$ ou mais da renda familiar total. $N$ as famílias cuja renda dos adultos era menor do que 3 salários mínimos, as diferenças não eram significativas. $N$ as famílias cuja renda dos adultos situava-se entre 3 e 6 salários mínimos, $8 \%$ dos meninos e $28 \%$ das meninas que trabal havam não contribuíam para a renda familiar, enquanto $24 \%$ dos meninos e $7 \%$ das meninas respondiam por $25 \%$ ou mais da renda familiar total $(p=0,03)$. N as famílias com renda dos adultos superior a 6 salários mínimos, $11 \%$ dos meninos e $27 \%$ das meninas trabal hando não contribuíam para a renda familiar, enquanto nenhuma menina eapenas $1 \%$ dos meninos aportavam $25 \%$ ou mais da renda familiar total $(p=0,18)$.

$\mathrm{N}$ as famílias cuja renda dos adultos era menor do que 3 salários mínimos, $23 \%$ dos adolescentes não freqüentaram a escola no ano anterior à entrevista. Esta proporção reduzia-se a $16 \%$, quando a renda dos adultos situava-se entre 3 e 6 salários mínimos e chegava a 5\%, quando a renda dos adultos era superior a 6 salários mínimos $(p<0,001)$. Além disso, a contribuição de $25 \%$ ou mais da renda familiar total alcançava $46 \%$ dos adolescentes fora da escola e $27 \%$ daqueles que freqüentavam a escola $(p=0,10)$. Por outro lado, em famílias com renda dos adultos até 6 salários mínimos, jornadas de 40 ou mais horas semanais eram a realidade de metade das crianças e adolescentes que trabal havam. Quando a renda dos adultos era superior a 6 salários mínimos, esta condição alcançava 30\% dos trabalhadores menores $(p<0,01)$.

A contribuição de crianças e adolescentes com algum rendimento monetário para o pagamento de despesas (aluguel, luz, alimentos, roupas, saúde e educação), inicia-se a partir dos 10 anos de idade. Entre 10 e 13 anos de idade, cerca de $39 \%$ das crianças trabalhadoras contribuíam para o pagamento de despesas, proporção que sobe para $50 \%$ dos 14 aos 17 anos. M ensalmente, as crianças e adolescentes trabalhadores gastavam em média $R \$ 26,00$ com despesas próprias e da família e $25 \%$ gastavam mais de $R \$ 40,00$ com estas despesas. 0 gasto com despesas próprias era em média de 
Tabela 2

Contribuição infantil à renda familiar conforme a renda familiar dos adultos. Pelotas, RS, 1998.

\begin{tabular}{lccc}
\hline Contribuição Infantil $(n)$ & $\begin{array}{c}<3 \mathrm{sm} \\
(\mathrm{n}=150)\end{array}$ & $\begin{array}{c}\text { Renda Familiar dos Adultos } \\
3 \mathrm{a}<6 \mathrm{sm}\end{array}$ & $\begin{array}{c}>6 \mathrm{sm} \\
(\mathrm{n}=113)\end{array}$ \\
\hline Nada (49) & 10.7 & 13.3 & 15.4 \\
$<5 \%(66)$ & 6.0 & 15.9 & 33.3 \\
$5 \mathrm{a}<10 \%(68)$ & 13.3 & 12.4 & 29.1 \\
$10 \mathrm{a}<25 \%(103)$ & 22.7 & 38.9 & 21.4 \\
25 a 100\% (94) & 47.3 & 19.5 & 0.9 \\
Total (380) & 39.5 & 29.7 & 30.8 \\
\hline
\end{tabular}

p<0,000; sm = salários mínimos

$R \$ 16,00$ e $10 \%$ gastavam acima de $R \$ 60,00$. A contribuição às despesas familiares era em média de $\mathrm{R} \$ 10,00$ e $10 \%$ gastavam acima de $\mathrm{R} \$ 39,00$. Dos 14 aos 17 anos, o gasto médio com despesas próprias era $\mathrm{R} \$ 21,00$ e com despesas familiares, $R \$ 13,00$, não havendo diferença de gênero.

Em média, as meninas contribuíam com $\mathrm{R} \$ 33,00$ para as despesas familiares e os meninos com $R \$ 27,00(p=0,30)$. A proporção de meninas (48\%) contribuindo para o pagamento de despesas familiares era significativamente maior do que a de meninos ( $35 \%)(p=0,02$; $R R=1,35 ;$ I $C 95 \%=1,1-1,7)$. Dos 14 aos 17 anos de idade, esta contribuição alcança $53 \%$ das meninas e $41 \%$ dos meninos $(p<0,05)$.

A proporção de menores trabalhadores contribuindo para as despesas familiares decrescia linearmente à medida que aumentava a renda familiar dos adultos $(p<0,001)$ (Tabela 3). Além disso, o valor monetário médio da contribuição infantil às despesas familiares era de $R \$ 36,00$ para famílias com renda dos adultos menor de 3 salários mínimos; $R \$ 30,00$ para famílias com renda dos adultos entre 3 e 6 salários mínimos e $\mathrm{R} \$ 24,00$ para famílias com renda dos adultos maior de 6 salários mínimos ( $\mathrm{pTL}=0,09$ ).

0 trabalho infantil significou uma importante contribuição financeira à economia dos bairros estudados. As 388 crianças e adolescentes trabal hando e recebendo total izavam $R \$ 31.641,00$ mensais, ou $R \$ 379.692,00$ anuais. Os 22 setores censitários estudados representavam cerca de $10 \%$ do total de setores censitários urbanos de Pelotas. M antendo-se a mesma proporção de crianças e adolescentes na faixa etária estudada e uma prevalência de trabalho infantil de $10 \%$, estima-se que na cidade existam cerca de 50.000 indivíduos entre 6 e 17 anos, talvez 4.760 trabalhando e 3.880 auferindo al gum ganho monetário com seu trabal ho. Assim, mantendo-se a renda média identificada para a amostra, a contribuição anual do trabal ho infantil à economia da cidade seria de aproximadamente $\mathrm{R} \$ 3.796 .920,00$.

\section{Discussão}

0 estudo caracterizou o trabal ho infantil em uma ampla amostra (4.924) de indivíduos entre 6 e 17 anos de idade, fato ainda raro em estudos sobre a temática no Brasil e no mundo (Committee on the Health and Safety Implications of Child Labor, 1998; Graitcer et al., 1998). As perdas foram de $7,6 \%$, valor bastante razoável para estudos epidemiológicos, especialmente considerando as dificuldades particulares do problema em estudo (Kleinbaum et al., 1982). M etodologicamente, os achados mostram boa validade interna, reforçada pelo rigor no planejamento e condução do trabalho de campo (Rothman et al., 1998).

A comparação entre nossos achados e aqueles do Brasil, de outros países e do mundo é bastante desafiadora, devido à falta de dados similares e às diferenças entre os grupos estudados, como por exemplo em idade, atividades econômicas e contexto social. A rel evância da comparação impõe, entretanto, uma análise tentativa. Os achados que podem ser plenamente generalizados para a população residente em bairros populares de Pelotas, também são referências úteis para estudos em outras regiões urbanas do país e do exterior.

A prevalência total de $10 \%$ de trabal ho infantil em Pelotas (de 6-17 anos) foi cerca de 
$40 \%$ menor do que a freqüência nacional (25\% de 10 a 17 anos), (Fausto et al., 1996), também sendo inferior à proporção observada em populações de 5 a 17 anos em todo o mundo (23\%), em países da América Latina e Caribe (20\%) e da Ásia e Pacífico (25\%) (Facchini et al., 2003; Forastieri, 1997; ILO, 1998 e 2002).

Entre os trabalhadores mais jovens, a prevalência de trabalho infantil em Pelotas ( $1,5 \%$ de 6-9 anos) foi similar à prevalência das economias desenvolvidas ( $1,4 \%)$, e menor do que a encontrada na Ásia e em países do Pacífico (12,3\% de 5-9 anos), da América Latina (10,6\% de 5 a 9 anos), da África Subsaariana $(23,6 \%$ de 5-9 anos) e do mundo ( $12,2 \%$ de 5-9 anos), (Forastieri, 1997; ILO, 1998 e 2002).

A prevalência de $8 \%$ de trabalho infantil de 10 a 14 anos de idade encontrada na população urbana brasileira foi similar àquela de nosso estudo ( $7 \%$ de 10 a 13 anos). M as, na população de Pelotas de 15 a 17 anos de idade a prevalência de trabalho infantil foi bastante mais baixa $(23,3 \%)$, em comparação a $31,3 \%$ em países desenvolvidos, $46,6 \%$ no Brasil, $35 \%$ na América Latina e Caribe e $42,4 \%$ no mundo (Fausto et al., 1996; Forastieri, 1997; ILO, 1998 e 2002).

A presença no trabalho dos meninos $(13,4 \%)$ foi significativamente maior do que a das meninas $(5,7 \%)$ em todas as faixas etárias. Estimativas prévias para o Brasil (Fausto et al.,1996) e o mundo (ILO, 1998) também mostravam uma maioria de meninos no trabalho infantil. Entretanto, a última estimativa da Organização Internacional do Trabalho (ILO, 2002) não mostrou diferenças significativas de gênero na prevalência mundial de trabalho infantil. As diferenças observadas em nossos achados e o de outros estudos são explicadas, pelo menos em parte, pelas altas taxas de desemprego que atingem de modo mais severo as mulheres, inclusive as mais jovens (Committee on the $\mathrm{H}$ ealth and Safety Implications of Child Labor, 1998; Graitcer, 1998).

0 trabalho infantil em Pelotas foi mais visível no comércio, serviços (domésticos e não domésticos) e construção civil, ramos cuja prevalência local foi maior que a nacional (Fausto et al., 1996). Quase metade (44,5\%) dos trabaIhadores infantis de Pelotas estava no setor de serviços. Este achado ésimilar ao de regiões urbanas brasileiras (45,9\%) (Fausto et al., 1996), mas superior à estimativa global (cerca de $10 \%)(I L O, 1998)$, que inclui crianças trabaIhando na agricultura (cerca de $70 \%$ dos traba-

\section{Tabela 3}

Contribuição de crianças e adolescentes trabalhadores às despesas familiares segundo a renda familiar dos adultos. Pelotas, RS, 1998.

\begin{tabular}{lcc}
\hline Renda dos adultos & $\begin{array}{c}\text { Contribuíam com as despesas } \\
\text { n }\end{array}$ \\
\hline M enos de 3 sm & 78 & 47.9 \\
3 a 6 sm & 49 & 40.5 \\
Mais de 6 sm & 34 & 27.4 \\
Total & 161 & 39.5
\end{tabular}

sm = salários mínimos, $p=0,001$

Ihadores infantis). Em Pelotas, o comércio foi o segundo tipo de trabalho mais freqüente $(34,0 \%)$, sendo maior do que 0 achado brasileiro para crianças urbanas (17,4\%) (Fausto et al., 1996), e a estimativa global (cerca de 8,3\%) (ILO, 1998).

A inserção ocupacional das meninas concentrou-se fortemente em comércio e serviços domésticos, enquanto os meninos mostravam maior dispersão, incluindo atividades altamente perigosas como construção civil eindústria. A pequena inserção na indústria (5\%) e a enorme presença no mercado informal (88\%) podem refletir os efeitos sobre o trabalho infanto-juvenil da desindustrialização, desemprego e estagnação da economia local nos últimos 20 anos (Graitcer et al., 1998). Em outros estudos, a crise econômica também tem se associado ao aumento da participação de crianças e adolescentes em atividades comerciais e como empregados domésticos, aumentando a presença destes trabalhadores no mercado informal, incluindo o comércio de rua (Lim, 2000).

As jornadas de trabalho de $40 \%$ das crianças e adolescentes de Pelotas eram de tempo completo (40 horas ou mais). Embora proporcionalmente menor do que a situação nacional (Fausto, 1996), este achado reforça o lado perverso do trabal ho infantil, devido aos riscos aumentados de exposição ocupacional e baixo aproveitamento escolar.

0 desemprego crescente e a estagnação econômica têm influências negativas sobre o salário dos trabal hadores (Committee on the H ealth and Safety I mplications of Child Labor, 1998; Graitcer et al., 1998). N este contexto, a renda mensal das crianças e adolescentes trabalhando foi relativamente baixa (média $=R \$ 72,00$ ), especialmente se comparada a regiões mais in- 
dustrializadas do país (Fausto et al., 1996). Além disso, nem todas as crianças trabalhadoras recebiam remuneração, mesmo trabal hando arduamente. Sob rótulos de ajuda doméstica, aprendizado ou treinamento para a função, esta situação era mais grave para as meninas, especialmente as mais jovens ( Fausto et al., 1996; Unicef, 1997).

É preciso cautela ao discutir a contribuição econômica de crianças e adolescentes, que geralmente ganham baixos salários e às vezes geram uma pequena proporção da renda familiar total (Graitcer et al., 1998; U nicef, 1997). Entretanto, esta contribuição pode ser bastante significativa nas famílias de mais baixa renda. Em Pelotas, a proporção da contribuição infanto-juvenil à renda familiar total crescia, quando a renda familiar dos adultos diminuía. Igualmente, quanto menor a renda familiar dos adultos, maior a exclusão escolar de adolescentes trabalhadores $(p<0,001)$ e maior a jornada de trabalho infanto-juvenil $(p<0,01)$. Assim, apesar das controvérsias sobre o elo entre pobreza e trabalho infanto-juvenil, (Fausto et al., 1996; Graitcer et al.,1998; Unicef, 1997) nossos achados mostraram que, em contextos urbanos de maior pobreza, as crianças trabal hadoras são mais jovens, são mais essenciais para o incremento da ren da familiar e correm um maior risco de não completar o ciclo escolar básico.

De modo geral, o trabal ho infanto-juvenil mostrou-se efetivo no incremento da renda familiar, aumentando-a em cerca de $9 \%$, se comparada à renda de famílias em que não havia a ocorrência de trabalho infantil. Os meninos trabalhadores são mais jovens, estão em maior proporção no mercado de trabalho e contribuem mais para a renda familiar. Em contraste, as meninas, especialmente as mais jovens, têm jornadas mai ores, inclusive noturna, recebem menos e contribuem proporcionalmente mais para o pagamento das despesas familiares. Portanto, a importância do trabalho infanto-juvenil para a família não pode ser medida apenas em unidades monetárias (Graitcer et al.,1998). Questões sociais, culturais e históricas contribuem para que desde jovens homens e mulheres tenham oportunidades de trabalho, remuneração e compromissos familiares diferentes.
A avaliação do número de crianças trabaIhando é o primeiro passo na identificação da "epidemia oculta" (Graitcer et al.,1998; U nicef, 1997), enquanto o reconhecimento do significado do trabalho infantil para a economia das famílias mais pobres é essencial para o estabelecimento de estratégias adequadas para a intervenção no problema. Portanto, há necessidade de novos estudos, que aprofundem a investigação da contribuição econômica do trabalho infantil na complexa rede de estratégias de sobrevivência familiar.

A análise estratificada do uso da força de trabalho infantil segundo a idade e o gênero mostrou-se útil para uma melhor delimitação e priorização do problema. Os ajustes macroeconômicos e as mudanças no mundo do trabaIho estão provocando empobrecimento das famílias, novas demandas do mercado de trabaIho, aumento de trabalhos pouco qualificados no setor de serviços e falta de fiscalização e cumprimento das leis, contribuindo para a transformação do significado do trabal ho infantil (Committee on the Health and Safety Implications of Child Labor, 1998; Graitcer et al., 1998). Os marcantes vínculos do trabalho infantil com pobreza, educação e gênero reforçam a urgência de um desenvolvimento econômico e social mais abrangente em nosso país.

Para uma adequada abordagem do problema necessita-se de uma agenda de pesquisas ousada e abrangente, capaz de melhorar a qualidade dos dados, impulsionar o monitoramento e a vigilância, compreender as implicações da natureza mutável do trabalho, das questões sociais e institucionais e identificar soluções inovadoras (Graitcer et al.,1998).

As interven ções para reduzir o impacto social e econômico do trabalho infantil precisam ser dirigidas tanto à redução da oferta e demanda de força de trabalho infantil, quanto à maximização dos esforços de promoção da saúde e da educação de crianças e adolescentes. Políticas efetivas de bem-estar social e redução da pobreza, investimentos em educação, desenvolvimento infantil integral, proteção de trabaIhadores adolescentes e a criação de ambientes sociais seguros e saudáveis deverão reduzir o ingresso de crianças e adolescentes no trabalho (Graitcer et al.,1998). 


\section{Agradecimentos}

Agradecemos 0 apoio recebido da Capes, CN Pq, Fapergs, Rede U nitrabalho, Organização Pan-Americana da Saúde e Fogarty Foundation que viabilizaram este estudo. Também agradecemos 0 apoio ao estudo e os comentários a este artigo de David Christiani (Occupational Health Program) e M ichael Reich (Takemi Program in International $\mathrm{H}$ ealth) da $\mathrm{H}$ arvard School of Public Health.

\section{Referências bibliográficas}

Anker R \& M elkas H 1995. Economic incentives for children and families intended to eliminate of reduce child labour. ILO, Geneva.

Comissão Estadual de Combate ao Trabalho Infantil no Rio Grande do Sul 1996. Diagnóstico preliminar sobre trabalho infanto-juvenil no Rio Grande do Sul. DRT - RS, Porto Alegre.

Committee on the Health and Safety Implications of Child Labor 1998. Protecting youth at work: health safety and development of working children and adolescents in the U nited States. National Research Council and Institute of M edicine, Washington.

Cruz N eto, O \& M oreira, MR 1998. Child and adolescent labor: factors, legal aspects, and social repercussions. Cadernos de Saúde Pública 14(2):437-441.

Dean JA et al. 1995. Epi Info, Version 6.02: a word-processing, database, and statistics program for public health. CDC, Atlanta, Georgia.

Facchini LA et al. 2003. Individuals at risk: the case of child labor, pp. 52-71. In J H eymann (org.). Global inequalities at work. Oxford, N ova York.

Fassa, AG et al. 2000. Child labor and health: problems and perspectives. International Journal of O ccupational and Environmental Health 6(1):55-62.

Fausto A \& Cervini R 1996. 0 trabalho e a rua: criançase adolescentes no Brasil urbano dos anos 80. Cortez, São Paulo.

Forastieri V 1997. Children at work: health and safety risks. International Labour Office, Geneva.

Graitcer PI \& Lerer LB 1998. Child labor and health: quantifying the global health impacts of child labor. The World Bank, Washington.

Himes J et al. 1994. Child labour and basic education in Latin America and the Caribbean: a proposed U NICEF initiative. Unicef, Florença, Itália.

IBGE (Instituto Brasileiro de Geografia e Estatística) 1992. Censo demográfico 1991: resultados preliminares. IBGE, Rio de Janeiro.
IBGE (Instituto Brasileiro de Geografia e Estatística) 2001. Pesquisa N acional por Amostra de Domicílios - PNAD 1999. IBGE, Rio de Janeiro.

ILO (International Labor Organization) \& IPEC (International Program on the Elimination of Child Labor) 2000. Investigating child labour guidelines for rapid assessment. ILO, Geneva.

ILO (International Labour Organization) 1998. Statistics on working children and hazardous child labour in brief. ILO, Geneva.

ILO (International Labour Organization) 1999. Convention 182: convention concerning the prohibition and immediate action for the elimination of the worst forms of child labor. ILO, Geneva.

ILO (International Labour Organization) 2002. Every child counts: new global estimates on child labour. ILO, Geneva.

Kleinbaum D et al. 1982. Epidemiologic research: principles and quantitative methods ( $2 a$ ed.). Wiley, John \& Sons, N ova York.

Lim JY 2000. The East Asian crisis and child labour in the Philippines. ILO, Geneva.

Rothman KJ \& Greenland S 1998. M odern epidemiology. Lippincott-Raven, Filadélfia.

Scanlon TJ et al. 2002. Child labour. British M edical Journal 325(7.361):401-403.

SPSS Incorporation 1997. SPSS for Windows: statistical package for the social sciences release 8.0. SPSS Inc., Chicago.

Unicef (The U nited Nations Children's Fund) 1996. The state of the world's children 1996: making child rights constitutional in Brazil. Unicef, Nova York.

Unicef (The United Nations Children's Fund) 1997. The state of the world's children 1997. Oxford U niversity Press, Oxford.

Artigo apresentado em 15/8/2003

Aprovado em 16/10/2003

Versão final apresentada em 29/11/2003 